\title{
Rest-to-Rest Motion of an Experimental Flexible Structure subject to Friction: Linear Programming Approach
}

\author{
Rajaey Kased and Tarunraj Singh \\ kased@eng.buffalo.edu tsingh@eng.buffalo.edu \\ State University of New York at Buffalo, Buffalo, NY 14260
}

\begin{abstract}
A linear programming approach designed to eliminate the residual vibration of the two-mass harmonic system subject to friction and undergoing a point-to-point maneuver is implemented. Techniques for non-robust and robust open loop controller design are explored. It is shown that consistent results can be obtained from experiments and the robustness against frequency uncertainty results in reduction in residual vibration as well as steady-state error.
\end{abstract}

\section{INTRODUCTION}

Linear programming (LP) is a powerful numerical optimization technique that is able to handle hundreds of constraints efficiently. It requires all constraints and costs to be linear in the unknown variable. This restriction on the use of LP may seem to eliminate practical use on real engineering problem. It is possible, however, to linearize some complex nonlinear systems to fit the required form of LP [1]. It is also possible to manipulate the original problem definition to fit the LP format as is done in [2], where a nonlinear constraint due to fuel usage is re-written as two linear constraints.

In system dynamics, LP can be used on linear discrete systems for time optimal and fuel limited time optimal control [3], [4], [2], where constraints linear in the discrete input sequence $u(k)$, are imposed to result in an input profile.

The significance of friction to the control community is in its effects on positioning systems and velocity tracking operation. Positioning applications include telescopes, antennas, machine tools, disk drives and robot arm positioning. Velocity control is also relevant in machine tool, disk drive and robot arm industrial applications which require the accurate tracking of a pre-determined trajectory. The effect of friction becomes accentuated in the low velocities region near the reference position.

The majority of work done on control of frictional systems is on rigid body systems. Yang and Tomizuka [5] exploited a simple relationship between a pulse input and the displacement of the rigid body. This utilizes the fact that the rigid body subject to a pulse input never changes the sign of the velocity and thus the Coulomb friction acts like a bias input. This scheme, known as Pulse Width Control (PWC), is presented in an adaptive control setting where an estimate of the friction is determined in real-time. Wijdeven and Singh [6], modified the PWC approach to increase accuracy in actual discrete implementation of the input. Their technique modulates the pulse height to compensate for a rounded up pulse width and is called Pulse Amplitude Pulse Width Control (PAPWC).

Additional schemes developed for rigid body systems include internal-model following error control [7], PID and state feedback linearization control [8] and variable structure control in order to try to handle qualitatively different friction regimes [9], [10]. Nonlinear PID control has also been developed to overcome the stick-slip behavior of friction [11].

Overcoming friction in flexible systems has been sparingly studied. This is despite the fact that the practical uses of controllers in this area include high precision overhead robot arms subject to friction at the joints, high-density hard-disk drives requiring the modelling of the system as well as the motion arm friction, and high-precision satellite pointing, where the friction effect is noticeable near the reference point.

Rathbun et al. [12] use the PWC control developed for the rigid body directly on the flexible system while ensuring stability by bounding the control gain. The system will however, result in undesired vibration near the reference point. Hamamoto et al. [13] use iterative feedback tuning to try to control the two mass harmonic system under the influence of friction. Two controllers (feedback and feedforward) are designed in order to account for the friction and reduce steady-state vibration.

A more recent approach poses the problem in a mixed integer linear programming setting, in order to accommodate for the friction sign change for the two-mass harmonic dynamics [14]. This is computationally expensive which precludes fine discretization of the maneuver time. Singhose et al. [15] use PD control on a second order system and input-shapers to try to compensate for Coulomb friction and eliminate vibration with positive velocity assumption.

This paper implements the technique developed by Kim and Singh [21], to eliminate the residual vibration of flexible structures subject to friction, undergoing point-to-point maneuvers. The proposed technique was illustrated on systems where the control input and frictional forces are collocated on an inertia. This approach uses linear programming to 
solve for optimal control profile which satisfy constraints that represent the dynamics of the system as well as positive velocity constraints.

\section{MATHEMATICAL Formulation}

The flexible structure that is used in this research is the two-mass harmonic oscillator subject to friction. The equations of motion are:

$$
\begin{aligned}
& {\left[\begin{array}{cc}
J_{1} & 0 \\
0 & J_{2}
\end{array}\right]\left[\begin{array}{l}
\ddot{\theta}_{1} \\
\ddot{\theta}_{2}
\end{array}\right]+\left[\begin{array}{cc}
\beta+c_{1} & -\beta \\
-\beta & \beta+c_{2}
\end{array}\right]\left[\begin{array}{l}
\dot{\theta}_{1} \\
\dot{\theta}_{2}
\end{array}\right]+} \\
& +\left[\begin{array}{cc}
k & -k \\
-k & k
\end{array}\right]\left[\begin{array}{l}
\theta_{1} \\
\theta_{2}
\end{array}\right]=\left[\begin{array}{l}
1 \\
0
\end{array}\right](u(t)-f(\underline{\dot{\theta}})) .
\end{aligned}
$$

The friction model $f(\underline{\dot{\theta}})$ is the classical static-Coulomb friction model:

$$
f(\dot{\theta})=\left\{\begin{array}{cl}
f_{c} \operatorname{sign}(\dot{\theta}) & \text { if } \dot{\theta} \neq 0 \\
f_{s} \operatorname{sign}\left(u_{\text {sum }}\right) & \text { if } \dot{\theta}=0 \text { and } u_{\text {sum }}>f_{s} \\
u_{\text {sum }} & \text { if } \dot{\theta}=0 \text { and } u_{\text {sum }} \leq f_{s} .
\end{array}\right.
$$

In the rest of the paper, the damping in the system is ignored in the controller design because the Coulomb friction dominates the nonconservative forces.

Since LP is an optimization technique, all equations of motion must be posed as constraints. Here, the cost function does not play a role in the solution since the LP is only used to satisfy a large number of constraints efficiently, to arrive at feasible control profiles.

In order to properly pose the LP problem, the states are re-written in discrete-time domain in terms of the state matrices and previous inputs (ARMA Model). Equation (3) represents the states of the system at $k=N+1$.

$$
x(N+1)=A^{N} x(1)+\sum_{k=1}^{N} A^{N-k} B u(k)
$$

The unknowns in equation (3) are the input sequences $u(k)$.

\section{A. System Subject to Friction}

Equation (3) can be reformulated to be written in block matrix form as

$$
x_{f}-A^{N} x(1)=\left[\begin{array}{llll}
A^{N-1} B & A^{N-2} B & \ldots & B
\end{array}\right]\left[\begin{array}{c}
u(1) \\
u(2) \\
\vdots \\
u(N)
\end{array}\right],
$$

where $x_{f}$ are the desired final states at $k=N+1$. Equation (4) is an equality constraint used in the LP that satisfies the dynamics of the system.

The number of constraints depend on the number of desired data points, $N$ and the sampling time $T$. The final maneuver time is given as $t_{f}=N T$. Assuming the number of samples stays the same, a reduction in the final time will result in a reduction in the sampling time of the system.

For a system under the influence of friction, positive velocity is assumed for the mass subject to friction and thus the $\operatorname{sign}(\dot{\theta})$ term in equation (2) becomes positive. The equations of motion are written similar to equation (3):

$x(N+1)=A^{N} x(1)+\sum_{k=1}^{N} A^{N-k} B u(k)-\sum_{k=1}^{N} A^{N-k} B f_{c}$.

The equality constraints can be written as

$$
\begin{gathered}
x_{f}-A^{N} x(1)+\sum_{k=1}^{N} A^{N-k} B f_{c}= \\
{\left[\begin{array}{llll}
A^{N-1} B & A^{N-2} B & \ldots & B
\end{array}\right]\left[\begin{array}{c}
u(1) \\
u(2) \\
\vdots \\
u(N)
\end{array}\right] .}
\end{gathered}
$$

In order to satisfy the positive velocity assumption, inequality constraints that represent positive velocity have to be included in the LP problem. These constraints, however, must be true at every time index, $k$. Therefore, a relationship between the output at any time $k$ and all previous input needs to be determined. A similar ARMA model can be written for the velocity of the system as in equations (3) and (5). Equation (7) represents the velocity constraints at each time index $k$.

$$
\begin{gathered}
{\left[\begin{array}{ccccc}
1 & 0 & \ldots & 0 & 0 \\
C B & 0 & \ldots & 0 & 0 \\
C A B & C B & \ldots & 0 & 0 \\
\vdots & \vdots & & \vdots & \vdots \\
C A^{N-2} B & C A^{N-2} B & \ldots & C B & 0
\end{array}\right]\left[\begin{array}{c}
u(1) \\
u(2) \\
u(3) \\
\vdots \\
u(N)
\end{array}\right] \leq} \\
{\left[\begin{array}{c}
C A x(1)-C B f_{c}-\epsilon \\
C A^{2} x(1)-C A B f_{c}-C B f_{c}-\epsilon \\
\vdots \\
C A^{N-1} x(1)-\sum_{i=1}^{N-1} C A^{N-1-i} B f_{c}-\epsilon
\end{array}\right]}
\end{gathered}
$$

The first entry in the inequality constraints states that the first input must be larger than stiction. The $\epsilon$ found in the velocity constraint equation is a numerically defined "zero" to accommodate for computer limited precision.

For a rigid body system subject to friction, the velocity of the mass will never change sign for positive pulse inputs. Therefore, it is reasonable to assume that multi-sign pulses may tend to force the velocity of the first mass to change sign more frequently than only positive pulses. Based on this assumption, the LP bounds on the inputs are given as

$$
0 \leq u(k) \leq U_{\max } \text { for } k=1,2, \ldots, N
$$

Equations (6), (7), and (8) constitute the LP problem, which is not used to minimize a specific cost per say, as much as it is used to find an input sequence that satisfies the constraints.

It is desired to obtain pulses as the LP solution of the input profile, to try to ensure that the input will always be greater than the stiction value, $f_{s}$. To force the LP solution to obtain a pulse input profile, the final time is reduced. The reduction of the final time will force the maneuver 
to end faster and this is done with input values that take their maximum possible value, $U_{\max }$. In order to obtain the minimum time possible for the LP to result in a feasible solution for $u(k)$, a bisection algorithm is used to reduce the final time efficiently.

\section{B. LP Desensitized Control}

The underlying presumption of controller design techniques that do not account of parameter variability, is exact knowledge of the true parameters of the system. In implementing a controller, the uncertainty in the system parameters will result in undesirable performance, which in the case of the two-mass harmonic oscillator, results in residual vibration at the end of the maneuver.

It is desirable to obtain a control profile that is insensitive to error in system parameter estimates. Liu and Singh [16] presented a technique that used the concept of sensitivity states which are defined as the derivative of the states with respect to the uncertain parameters. By forcing the sensitivity states to zero at the end of the maneuver, robustness to modelling errors is achieved. For the system under study, the uncertain parameter is taken to be the system stiffness, $k$.

The sensitivity states are defined as

$$
x_{1 s}=\frac{\partial x_{1}}{\partial k} \quad x_{2 s}=\frac{\partial x_{2}}{\partial k} .
$$

The states $x_{1}$ and $x_{2}$ are functions of the parameter $k$ and their sensitivities are required to be zero at the final time, $t_{f}$. Differentiating the equations of motion, equation 1 , with respect to $k$ results in

$$
\begin{aligned}
& J_{1} \ddot{x}_{1 s}+x_{1}+k x_{1 s}-x_{2}-k x_{2 s}=0 \\
& J_{2} \ddot{x}_{2 s}-x_{1}-k x_{1 s}+x_{2}+k x_{2 s}=0 .
\end{aligned}
$$

Placing equation (10) into matrix form

$$
\begin{gathered}
{\left[\begin{array}{c}
\ddot{x}_{1 s} \\
\ddot{x}_{2 s}
\end{array}\right]=} \\
-\left[\begin{array}{cccc}
1 / J_{1} & -1 / J_{1} & k / J_{1} & -k / J_{1} \\
-1 / J_{2} & 1 / J_{2} & -k / J_{2} & k / J_{2}
\end{array}\right]\left[\begin{array}{c}
x_{1} \\
x_{2} \\
x_{1 s} \\
x_{2 s}
\end{array}\right]
\end{gathered}
$$

reveals the relationship between the two equations. Using the elementary row operation $\mathcal{R}_{1} / J_{2}+\mathcal{R}_{2} / J_{1}$, equation 11 can be rewritten as

$$
\begin{aligned}
& {\left[\begin{array}{c}
\ddot{x}_{1 s} \\
\ddot{x}_{1 s} / J 2+\ddot{x}_{2 s} / J 1
\end{array}\right]=} \\
& -\left[\begin{array}{cccc}
1 / J_{1} & -1 / J_{1} & k / J_{1} & -k / J_{1} \\
0 & 0 & 0 & 0
\end{array}\right]\left[\begin{array}{c}
x_{1} \\
x_{2} \\
x_{1 s} \\
x_{2 s}
\end{array}\right]
\end{aligned}
$$

which shows that the two sensitivity equations of motion are not independent and only one is necessary to capture the dynamics of the two. From equation (12), the relationship between the sensitivity states are found to be

$$
\ddot{x}_{1 s}=-\frac{J_{2}}{J_{1}} \ddot{x}_{2 s} .
$$

The boundary conditions of the sensitivity states at the final time are

$$
x_{1 s} t_{f}=x_{2 s} t_{f}=\dot{x}_{1 s} t_{f}=\dot{x}_{2 s} t_{f}=0 .
$$

Integrating equation (13) twice and using the boundary conditions of the sensitivity states, the relationship between $x_{1 s}$ and $x_{2 s}$ is

$$
x_{1 s}=-\frac{J_{2}}{J_{1}} x_{2 s} .
$$

Combining equation (14) and the first equation in (10) results in the sensitivity state equation of motion that is augmented to the system state equation and results in the equation

$$
\ddot{x}_{2 s}=\frac{1}{J_{2}}\left(x_{1}-x_{2}\right)-\left(\frac{k}{J_{1}}+\frac{k}{J_{2}}\right) x_{2 s}
$$

The new equations of motion in continuous domain are

$$
\begin{gathered}
{\left[\begin{array}{ccc}
J_{1} & 0 & 0 \\
0 & J_{2} & 0 \\
0 & 0 & 1
\end{array}\right]\left[\begin{array}{c}
\ddot{\theta}_{1} \\
\ddot{\theta}_{2} \\
\ddot{\theta}_{1 s}
\end{array}\right]+} \\
+\left[\begin{array}{cccc}
k & -k & 0 \\
-k & k & 0 \\
-\frac{1}{J_{2}} & -\frac{1}{J_{2}} & \frac{k}{J_{1}}+\frac{k}{J_{2}}
\end{array}\right]\left[\begin{array}{c}
\theta_{1} \\
\theta_{2} \\
\theta_{1 s}
\end{array}\right]=\left[\begin{array}{l}
1 \\
0 \\
0
\end{array}\right]\left(u-f_{c}\right)(16)
\end{gathered}
$$

The variables shown in the current development are replaced with ones that are consistent with ones in the previous sections. The discrete-time state space equations can be derived from equation (16) and used in the same LP formulation as in Section II-A.

\section{EXPERIMENTAL SETUP AND RESUlts}

\section{A. Hardware, Software, System Identification}

An experimental testbed of a two-mass harmonic oscillator subject to friction is built and the proposed controllers are implemented. A figure of the experiment is shown in Figure 1.

The motor that is used is the MircoMo $4490024 B$ model, with a recommended no load torque operation of 0.192 [N$\mathrm{m}]$. The nominal torque that is used throughout this thesis is approximately $3.5 \times 10^{-3}[\mathrm{~N}-\mathrm{m}]$. This was calculated for an inertial load of $2.75 \times 10^{-4}\left[\mathrm{~kg}-\mathrm{m}^{2}\right]$ and a max velocity of approximately 2 [rev/s] reached in 1 [sec] (ie. $\alpha=2 \mathrm{rad} / \mathrm{sec}^{2}$. This torque value is used as a reference point for nominal torque values and is not a restrictive bound. This brush-less type motor is chosen over its ironcore counterpart due to friction considerations. The ironcore motors typically use a gear box to achieve similar torque values and thus the friction is increased.

The corresponding amplifier that is as used is a MircoMo MVP2001 A01 Driver Electronics $(M V P)$. The motor is 


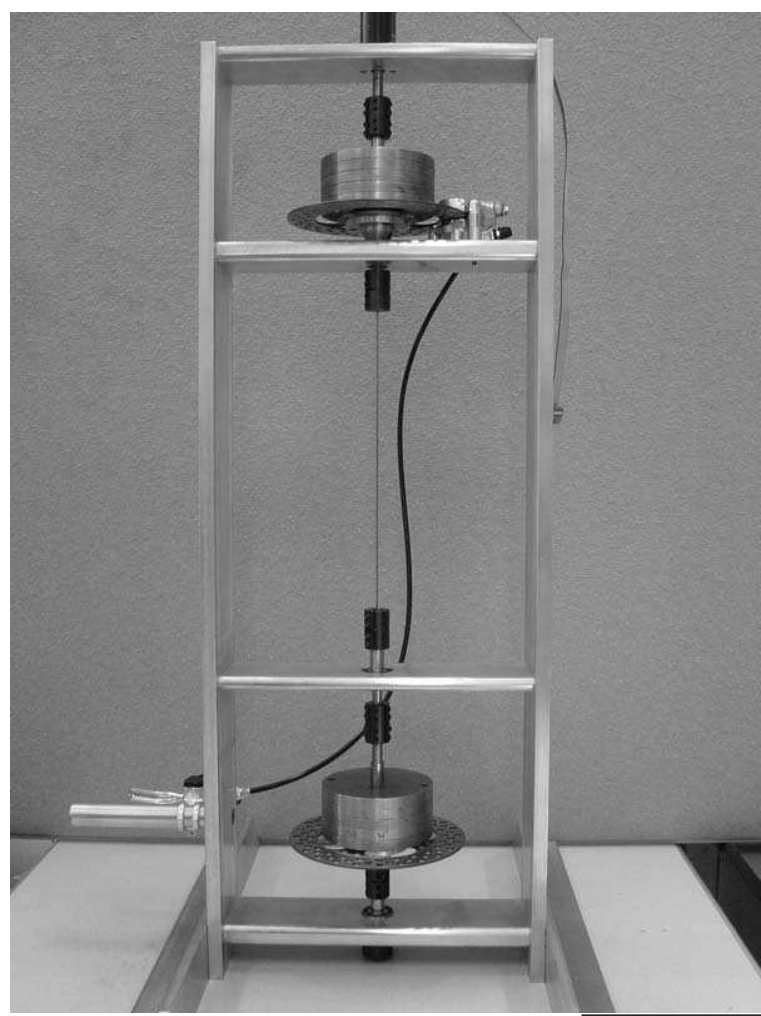

Fig. 1. Two Mass-Spring Harmonic Oscillator Subject to friction

commanded via serial commands to the $M V P$. The dynamics of the electronics of motor ( $M V P$, magnetic fields, etc.) are assumed to be negligible. An identification of the Amplifier and Motor shows that a model of a rigid body system subject to damping can be fit reasonably well. Therefore, the inertia and damping of the motor and amplifier can be lumped with that of the rotating mass. Two USDigital E6S series encoders with 2048 cycles per revolution quadrature (4 pulses per cycle) output are used to query the position of both masses. A Formula Evoluzione 9.5 Disk Brake Set was used to mount the inertias and apply the desired friction.

LabVIEW $^{1}$ is used as the real time data processing software for all the experiments ([17], [18]). MatLAB ${ }^{2}$ is used for all pre and post-processing (i.e. trajectory constructions, optimizations, etc.) of data and processing the results [19].

Through extensive system identification of the apparatus, the system parameters are estimated and are shown in Table I.

\section{B. Experimental Results}

The LP solutions for the non-robust and robust approach are shown in Figures 2(a) and 2(b). The sampling time used in the LP was .001 [s] in order to obtain an accurate input profile. With a smaller sampling time a profile closer to

\footnotetext{
${ }^{1}$ LabVIEW is a registered trademark of National Instruments, Inc.

${ }^{2}$ MatLAB is a registered trademark of The MathWorks, Inc., Natick, Mass.
}

\begin{tabular}{|c|c|}
\hline System Variable & Estimated Value \\
\hline$\hat{J}_{1}\left[\mathrm{~N}-\mathrm{m}-\mathrm{s}^{2}\right]$ & $1.35 \times 10^{-5}$ \\
\hline$\hat{J}_{2}\left[\mathrm{~N}-\mathrm{m}-\mathrm{s}^{2}\right]$ & $7.13 \times 10^{-5}$ \\
\hline$\hat{k}\left[\frac{\mathrm{N}-\mathrm{m}}{\mathrm{rad}}\right]$ & $1.00 \times 10^{-2}$ \\
\hline$\hat{f}_{c}[\mathrm{~N}-\mathrm{m}]$ & $4.32 \times 10^{-4}$ \\
\hline$\hat{f}_{s}[\mathrm{~N}-\mathrm{m}]$ & $1.58 \times 10^{-3}$ \\
\hline
\end{tabular}

TABLE I

EXPERIMENTAL PARAMETERS OF TWO-MASS HARMONIC OSCILLATOR

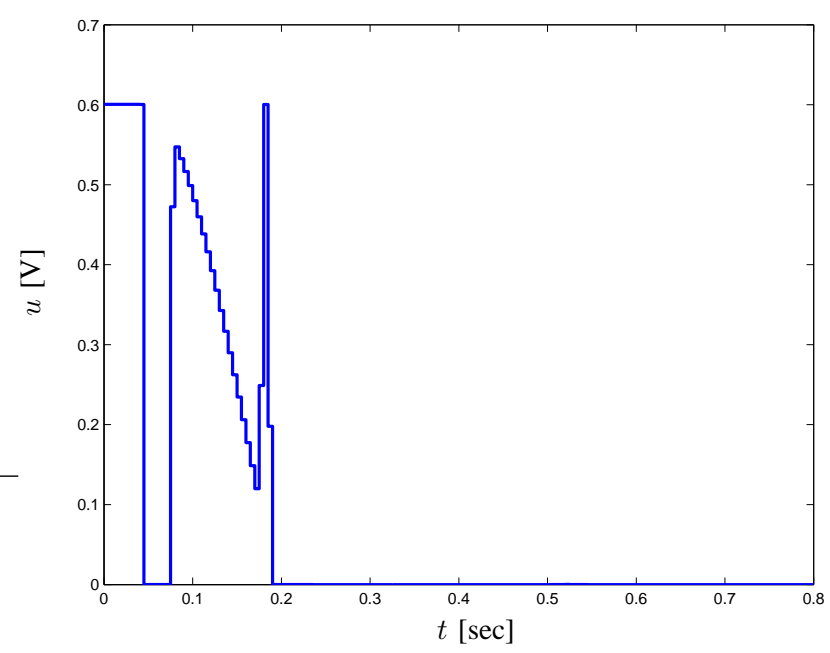

(a) Input profile from LP Non-Robust solution

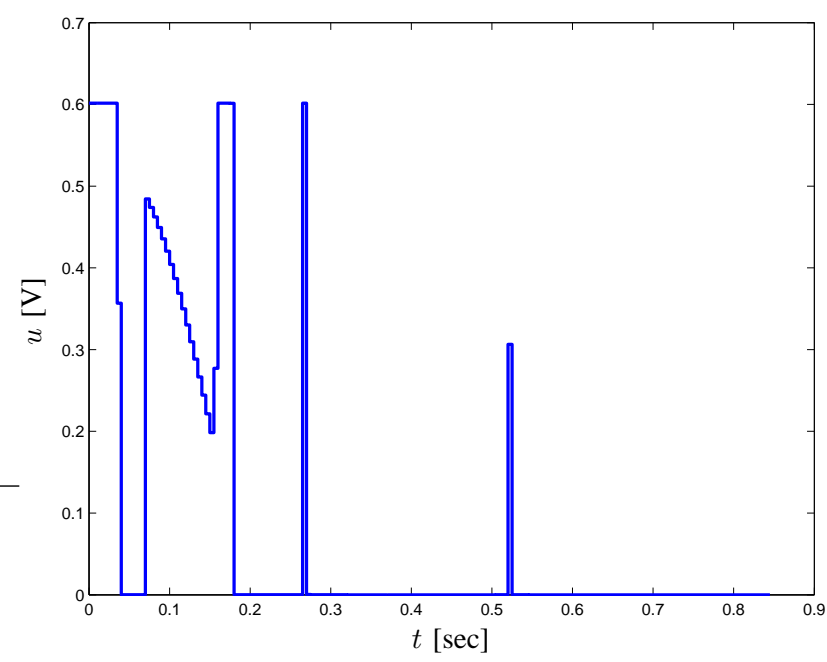

(b) Input profile from LP Robust solution

Fig. 2. LP Solution for Non-Robust and Robust Approaches

the "optimal" profile is obtained. Smaller sampling times will allow for finer choice of final times, in turn resulting in more precise choice of switching times and pulse height values. Since the sampling time in LabVIEW, however, is only .005 [s], every 5 points is extracted from the LP results. The desired position is $x_{f}=1[\mathrm{rad}]$ and the maximum 
input value, $U_{\max }$, is taken to be $3.8 \times 10^{-3}[\mathrm{~N}]$ which corresponds to $0.60[\mathrm{~V}]$.

The linearly decreasing region in Figures 2(a) and 2(b), are where the simulation shows the first mass as being stuck. In order to satisfy the positive velocity constraints, the LP solution provides a spring compensation force to ensure that the first mass does not change direction.

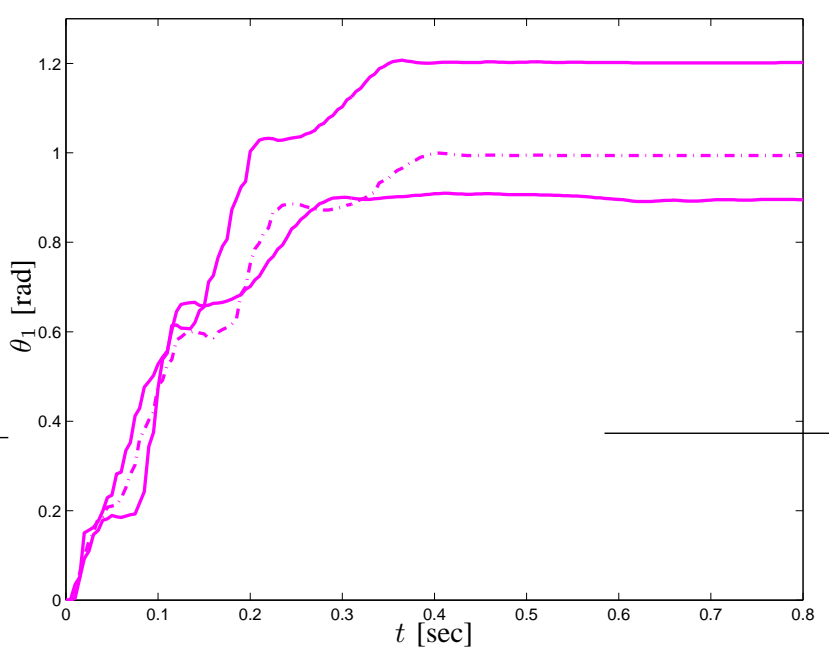

(a) First Mass Responses

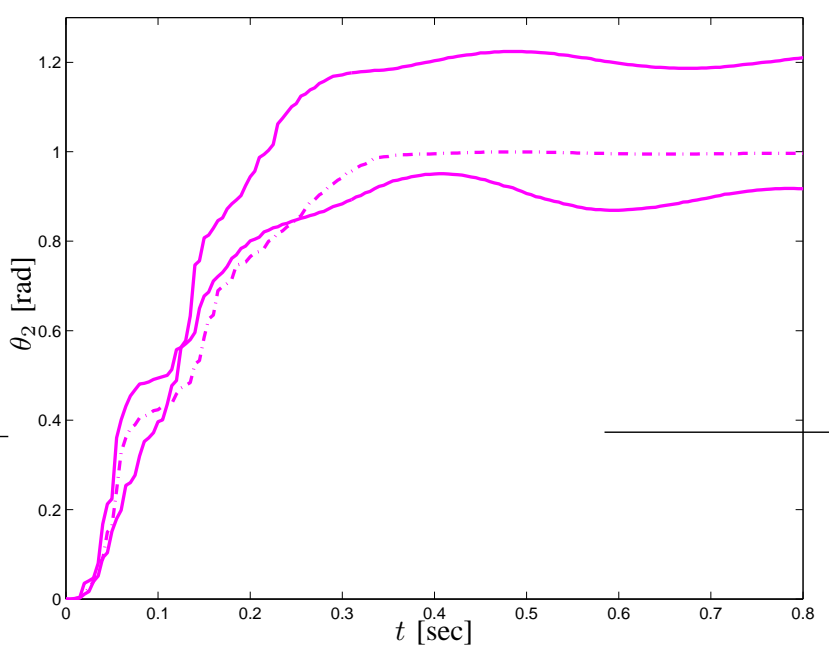

(b) Second Mass Responses

Fig. 3. The max and min steady-state errors of the LP Two Mass experimental results.

Twenty-five experiments are performed with the resulting input profiles. Figure 3 shows the extreme experimental results obtained using the non-robust input profile shown in Figure 2(a) for the first and second mass respectively. The steady-state values range from $0.9 \leq x_{d} \leq 1.2$ [rad], approximately -10 to $20 \%$. The middle trajectory corresponds to the "best" obtained trajectory. The extreme results shown for the first and second mass are for the same experiments (i.e. the max steady-state error for the first and second mass come from the same experiment). It should be noted that for larger steady-state errors of the first mass, which get stuck before the second mass comes to rest, corresponds to larger vibration of the second mass. This indicates a strong coupling between the estimate of friction and the residual vibration of the system.

The extreme cases also result in large residual vibration which are due to slight deviations in friction. The way

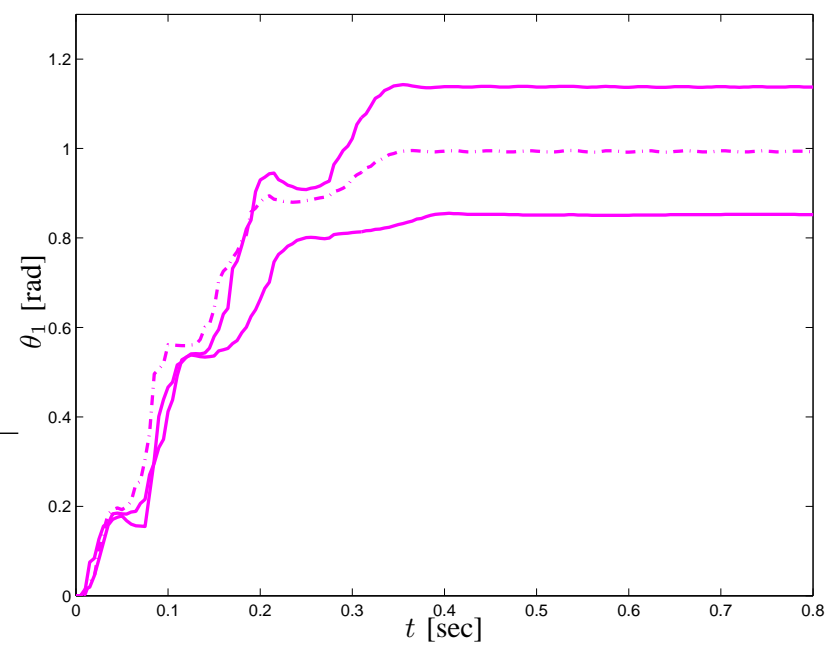

(a) First Mass Responses

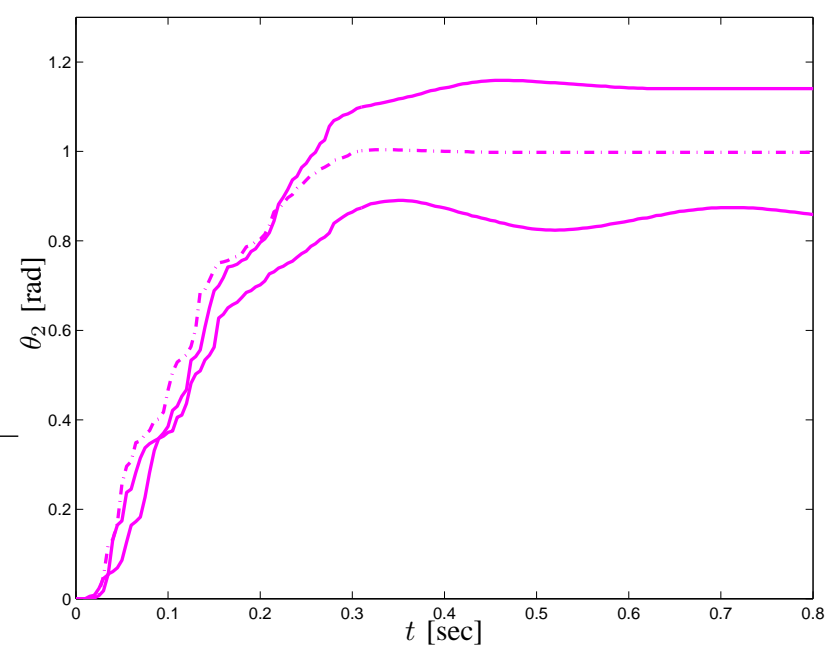

(b) Second Mass Responses

Fig. 4. The max and min steady-state errors of the Robust LP Two Mass experimental results.

the friction is set up in the experiment, slight warpage in the disk will result in different normal forces on the inertia. In general this type of nonlinearity is inherent to experimentation. This must be ignored, however, to simplify analysis and controller design. Despite the simplifications made, an understanding of the "true" system is necessary to explain experimental results.

Throughout the 25 experiments, the first mass trajectory occasionally has a slight change in sign in velocity. This is mainly attributed to friction uncertainties and nonlinearities. 
In the LP development, the friction is assumed to be constant and known as is seen in equation (5). However, the velocity sign change is minor enough that the LP solution is still effective. It is important to point out, that the effectiveness in the controller, despite the assumptions made, is reasonable for the open loop. The position of the second mass also shows that the vibration is cancelled by the end of the maneuver.

Figure 4 shows the worst and best experimental results for the robust case. Again, the assessment is based on steadystate error which is directly correlated to residual vibration of the second mass. Just as in the non-robust case, the plots for the maximum and minimum steady-state errors for both the first and second mass come from the same experiment.

Comparing Figure 4 to Figure 3 , it is seen that the worst case scenarios have improved for the robust case to resulting in less oscillation for the duration of the maneuver. There is also less variation across the experiments. This is

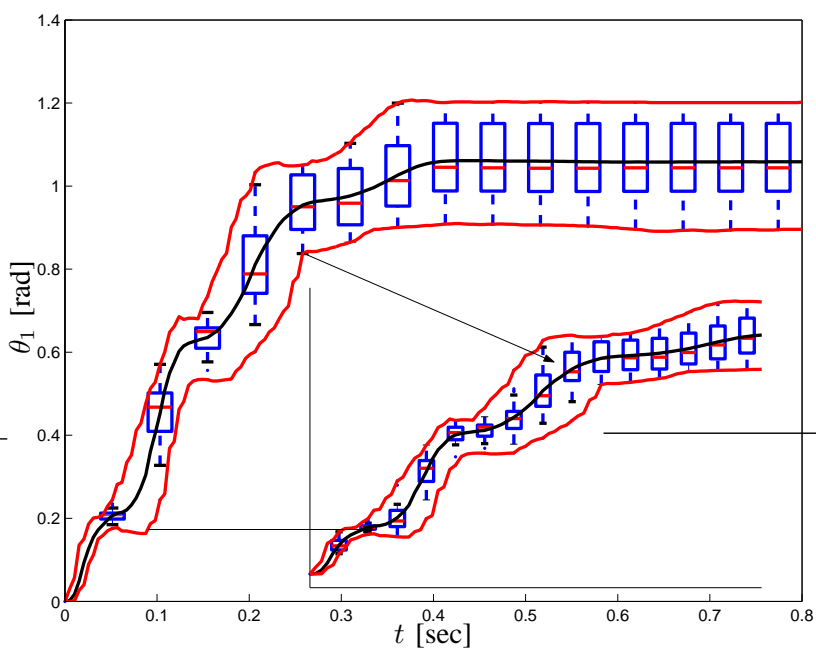

(a) First Mass Responses

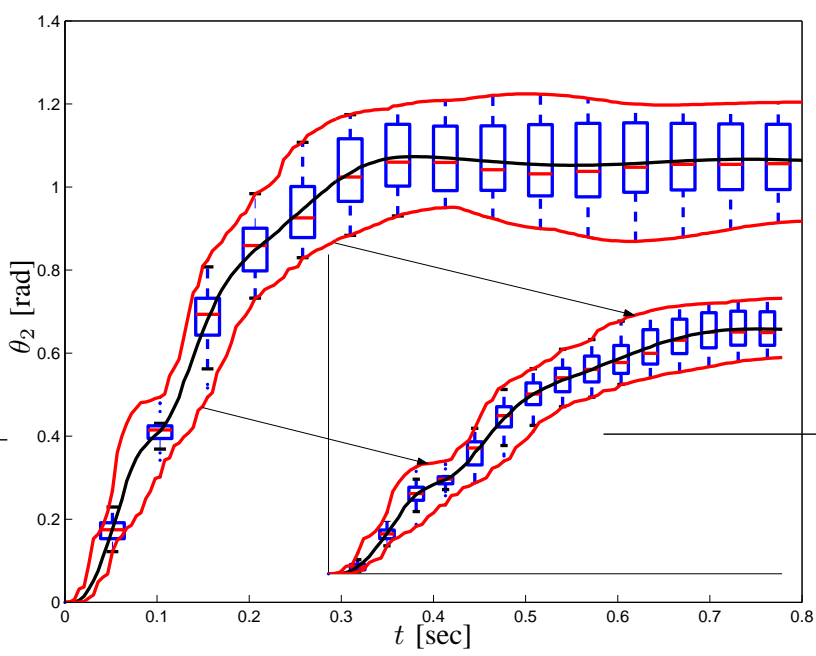

(b) Second Mass Responses

Fig. 5. Box plot distribution of point-by-point LP Two Mass experimental results. most clearly seen in the first 0.2 [s] of the experimental results. The non-robust results have more overlap between the worst case results, as opposed to the robust case where the trajectories are relatively close to each other. Also, it is seen that the amplitude of oscillation for the worst cases are less than the non-robust counterparts.

Another significant improvement in the robust result is that overall, the steady-state error has decreased. It has decreased to about $\pm 10 \%$ from the previous $\pm 10-20 \%$. The robust controller was designed to be insensitive to variation in the spring constant, $k$ which is related to the natural frequency of the system. Therefore it is expected that vibration near the reference point will be less than that of the non-robust formulation, as is the case. This decreased band of oscillation decreases the range for the first mass to get stuck in, at the end of the maneuver, thus decreasing the steady-state error.

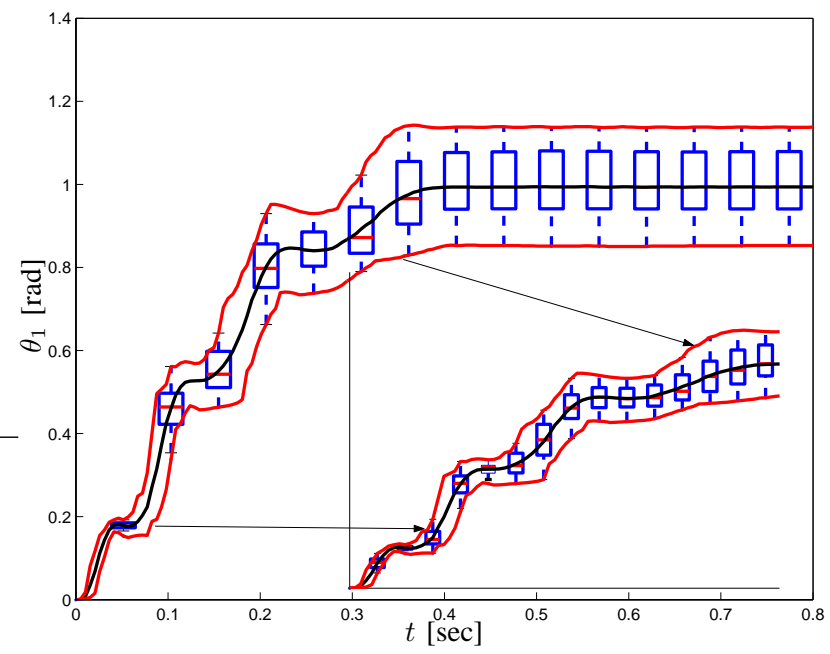

(a) First Mass Responses

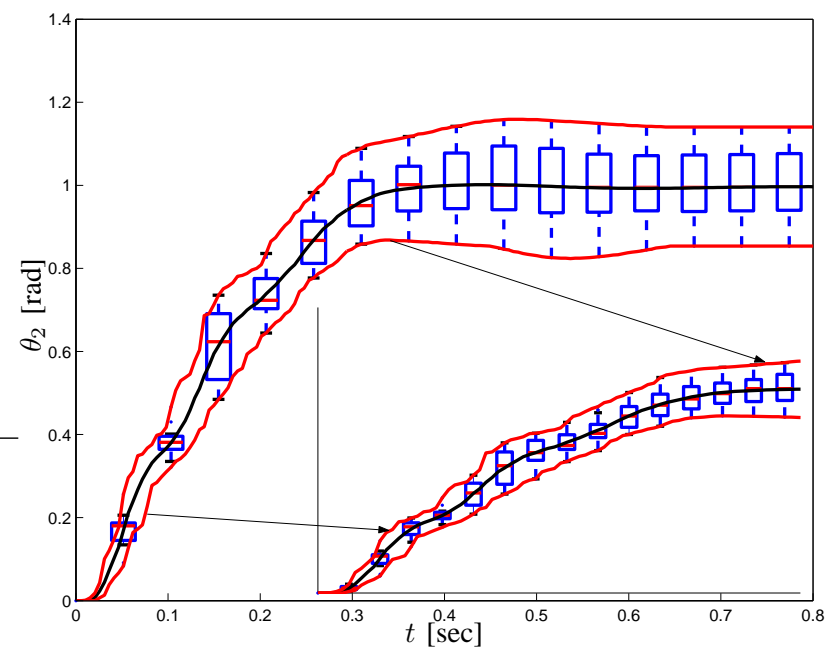

(b) Second Mass Responses

Fig. 6. Box plot distribution of point-by-point Robust LP Two Mass experimental results. 
In order to display the repeatability of the experiment, the distribution of the 25 experiments are displayed with a "Box-and-Whisker" distribution plot, mainly used in descriptive statistical analysis [20]. The box-and-whisker plot is represented by 5 numbers in the sorted data set: the minimum, first quartile, median, third quartile, and maximum value. It is a convenient way of showing the deviation of the entire data set from these points. The outlying data points are not considered when determining a minimum and maximum, but they are used to determine the quartiles and are shown as single dots in the plot that are placed beyond the whiskers.

Figure 5 illustrates the box plot distribution for the first and second mass across all experiments of the non-robust case. The middle trajectory represents the mean value of all experiments for each time $k T$. The bounding curves are the $\max$ and min of all experiments for each time $k T$. None of these curves represent an actual experimental trajectory. Each box plot represents the distribution of all experiments for that particular time. For the initial motion of the masses, a finer distribution is shown in an inset, to highlight the performance in that region.

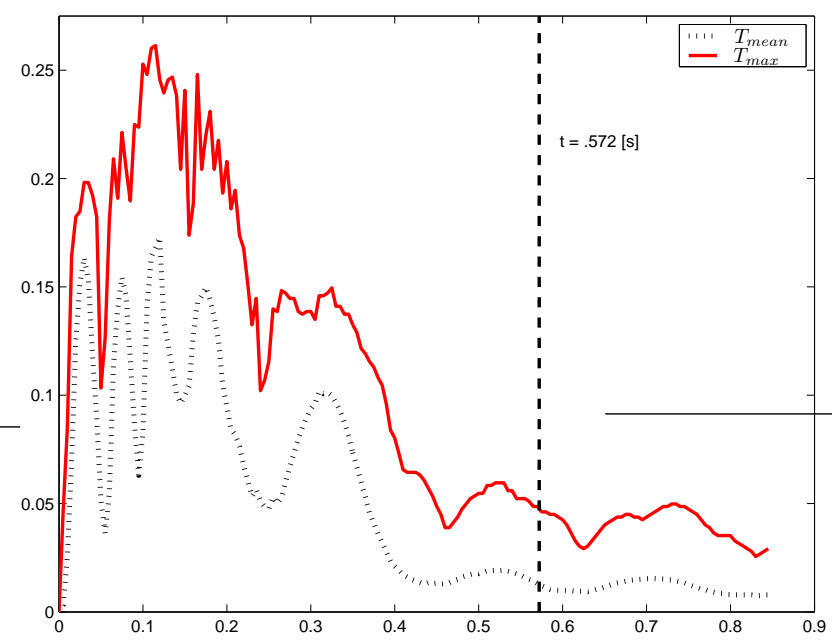

Fig. 7. Max and Mean Torsional Spring force due to relative displacements for 25 experiments (Non-Robust Controller Design)

The general trend in the range of the distribution is that it increases as position and velocity increase. It is expected that the point distribution will be larger as the mass increases position because of the friction variation around the inertia on which the friction force is applied. It is also expected that increased variation will occur at points where the motor applies its torque due to slight timing differences and controller saturation that may be present during pulse commands.

The improvement in the robust solution is further emphasized in the box-and-whisker evolution plot seen in Figure 6. The evolution of the positions generally have less variation across experiments. The same trends of increased variation is illustrated as in Figure 5 for the non-robust formulation.
A further comparison between the robust and non-robust solution is seen in Figures 7 and 8. The max and mean magnitude of the torsional spring force across all 25 experiments are plotted for both the non-robust and robust controllers respectively. It is seen that the maximum torsional spring force for the robust design is approximately $20 \%$ lower than the non-robust case. Furthermore, the spring force has been reduced throughout the entire maneuver.

This result is achieved by an increase in the number of pulses in the robust solution shown in Figure 2(b). The movement of the first mass towards the desired position is directed by the input pulses (i.e. four ramp up periods due to four pulses) as opposed to the non-robust case where the final ramp up is due the spring torsional force (i.e. three ramp up periods only two pulses). This is seen by comparing the pulse times in Figures 2(a) and 2(b) with the evaluation of the positions of the inertias in Figures 3 and 4 .

The times shown in Figures 7 and 8 are the times where the systems were designed to have completed the maneuver. Comparing the mean torsional spring magnitude for the

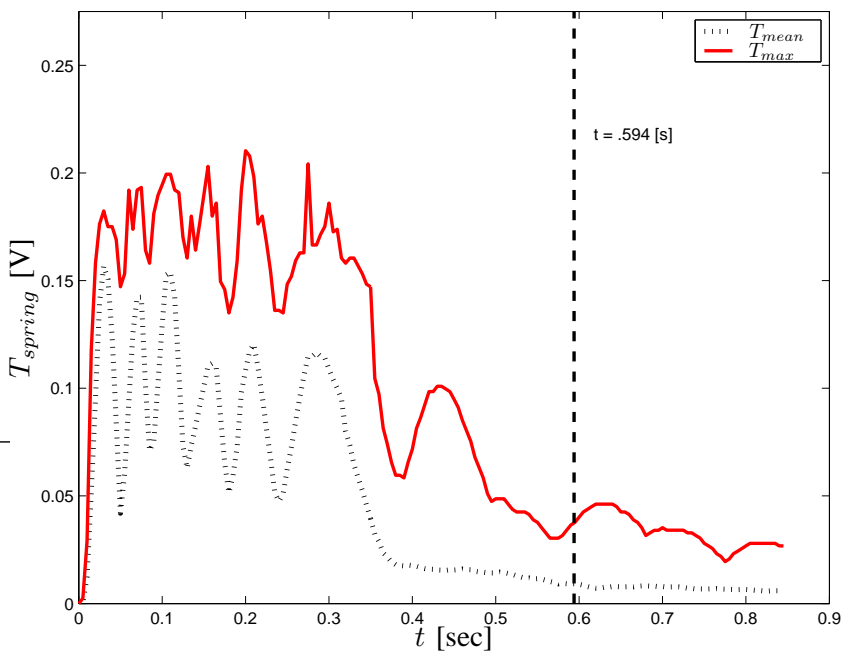

Fig. 8. Max and Mean Torsional Spring force due to relative displacements for 25 experiments (Robust Controller Design)

non-robust and robust controller design shows that there is residual energy in the system after the designed final time $t=.572[\mathrm{~s}]$ in the non-robust approach. The oscillations indicate a transfer of energy of the masses from potential to kinetic. The oscillations of the robust approach are reduced as compared to the non-robust approach showing a reduction in residual vibration in the system at the end of the maneuver.

\section{Conclusions}

A Linear Programming (LP) technique based on Kim and Singh's work [21], was implemented on a two-mass harmonic oscillator subject to friction for the robust and non-robust case. This technique only requires the user to 
select the sampling time. The LP approach automatically selects input sequence values at every sample in the interval. Reducing the final time forces the LP result to be pulses. The robust solution shows reduction in the steady-state error and residual vibration compared to the non-robust LP approach.

\section{ACKNOWLEDGMENT}

The authors would like to thank Formula ${ }^{3}$ for their kind donation of the hydraulic disk brake set used to mount the inertias and apply the friction.

\section{REFERENCES}

[1] Garret N. Vanderplaats. Numerical Optimization Techniques For Engineering Design with Applications. McGRAW-Hill Series in Mechanical Engineering. McGraw-Hill, Inc., 1984.

[2] Brian J. Driessen. On-Off Minimum-Time Control With Limited Fuel Usage: Near Global Optima Via Linear Programming. In Proceedings of the American Control Conference, pages 3875 - 3877. ACC, June 2000.

[3] G. Bashein. A simplex algorithm for On-Line computation of time optimal controls. IEEE Transactions on Automatic Control, 16:479482, 1971.

[4] S. Engell M. Kim. Speed-up of Linear Programming for TimeOptimal Control. In Proceedings of the American Control Conference. ACC, June 1994.

[5] S. Yang and M. Tomizuka. Adaptive pulse width control for precise positioning under the influence of stiction and coulomb friction. ASME Journal of Dynamic Systems, Measurements, and Control, 110:221-227, September 1988

[6] J.J.M. van de Wijdeven and Tarunraj Singh. Adaptive pulse amplitude pulse width control of systems subject to coulomb and viscous friction. In American Control Conference, June 2003. Denever, Colorado.

[7] Bong Jeun Kim and Wan Kyun Chung. Motion Control of Precision Positiong Systems Using Adaptive Compensation. In Proceedings of the American Control Conference, pages 4589-4594, 2002.

[8] F. Altpeter, P. Myszkorowski, M. Kocher, and R. Longchamp. Friction compensation: Pid synthesis and state control. In European Control Conference, page 1, 1997.

[9] Ruh-Hua Wu and Pi-Cheng Tung. Fast Pointing Control for Systems with Stick-Slip Function. ASME Journal of Dynamic Systems, Measurements, and Control, 126:614-626, 2004.

[10] H.G. Kwatny, C. Teolis, and M. Mattice. Variable structure control of systems with uncertain nonlinear friction. Automatica, 38:12511256, 2002.

[11] D. Neevel, B. Armstrong, and T. Jusik. New Results in NPID Control: Tracking, Integral Control, Friction Compensation and Experimental Results. In IEEE Trans. on Control Systems Technology, volume 9, pages 399-406, 2001.

[12] David B. Rathbun, Martin C. Berg, and Keith W. Buffinton. Pulse Width Control for Precise Positioning of Structurally Flexible Systems Subject to Stiction and Soulomb Friction. ASME Journal of Dynamic Systems, Measurements, and Control, 2003.

[13] K. Hamamoto, T. Fukuda, and T. Sugie. Iterative feedback tuning of controllers for a two-mass-spring system with friction. Control Engineering Practice, 11:1061-1068, 2003.

[14] Brian Driessen and Nader Sadegh. Minimum-time control of systems with Coulomb friction: near global optima via mixed integer linear progamming. Optimal Control Applications and Methods, 22:51-62, 2001.

[15] William Singhose, Jason Lawrence, and Keith Hekeman. An analytical solution for $\mathrm{z}$ zero vibration input shaper for systems with coulomb friction. In Proceedings of the American Control Conference, pages 4068-4073, 2002.

\footnotetext{
${ }^{3} \mathrm{http}: / /$ www.formula-brake.it
}

[16] Shin-Whar Liu and Tarunraj Singh. Robust Time-Optimal Control of Flexible Structures with Parametric Uncertainty. ASME Journal of Dynamic Systems, Measurements, and Control, 119:743-748, December 1997.

[17] J. Travis. LabVIEW For Everyone. National Instruments virtual instrumentation series. Prentice Hall, One Lake Street, Upper Saddle River, NJ, 2nd edition, 2002.

[18] R. H. Bishop. Learning with LabVIEW. Prentice Hall, One Lake Street, Upper Saddle River, NJ, 6i edition, 2001.

[19] The Math Works, Inc., 3 Apple Hill Drive Natick, MA 01760-2098. Optimization Toolbox For Use with MatLab, June 2001. Version 2.1.1 (Release 12.1) (online only.

[20] Ron Larson and Betsy Farber. Elementary Statistics: Picturing the World. Prentice Hall, $2^{\text {nd }}$ edition, 2003.

[21] T. Singh J.J. Kim. Controller design for flexible systems with friction: Linear programming approach. In Proceedings of American Control Conference. ACC, June 2003. 